\title{
Survival of pathogenic and lactobacilli species of fermented olives during simulated human digestion
}

\section{Francisco N. Arroyo-López ${ }^{1}$ *, Stéphanie Blanquet-Diot ${ }^{2}$, Sylvain Denis ${ }^{2}$, Jonathan Thévenot ${ }^{2,3}$, Sandrine Chalancon ${ }^{2}$, Monique Alric ${ }^{2}$, Francisco Rodríguez-Gómez ${ }^{1}$, Verónica Romero-Gil ${ }^{1}$, Rufino Jiménez-Díaz' and Antonio Garrido-Fernández ${ }^{1}$}

\author{
${ }^{1}$ Biotecnología de Alimentos, Instituto de la Grasa - Consejo Superior de Investigaciones Científicas, Seville, Spain \\ ${ }^{2}$ Centre de Recherche en Nutrition Humaine Auvergne, EA 4678, Conception Ingénierie et Développement de I'Aliment et du Médicament, Clermont \\ Université - Université d'Auvergne, Clermont-Ferrand, France \\ ${ }^{3}$ Centre de Recherche en Nutrition Humaine Auvergne, M2iSH, UMR INSERM/Université d'Auvergne U1071 USC-INRA 2018, Clermont Université - Université \\ d'Auvergne, Clermont-Ferrand, France
}

\section{Edited by:}

Fabio Minervini, Università degli Studi di Bari Aldo Moro, Italy

\section{Reviewed by:}

Chrysoula C. Tassou, Hellenic Agricultural Organization "DEMETER", Greece

Zhao Chen, Clemson University, USA

\section{*Correspondence:}

Francisco N. Arroyo-López,

Biotecnología de Alimentos, Instituto de la Grasa - Consejo Superior de Investigaciones Científicas, Avenida Padre García, Tejero n², 41012

Seville, Spain

e-mail: fnarroyo@cica.es
The present survey uses a dynamic gastric and small intestinal model to assess the survival of one pathogenic (Escherichia coli O157:H7 EDL 933) and three lactobacilli bacteria with probiotic potential (Lactobacillus rhamnosus GG, L. pentosus TOMC-LAB2, and L. pentosusTOMC-LAB4) during their passage through the human gastrointestinal tract using fermented olives as the food matrix. The data showed that the survival of the $E$. coli strain in the stomach and duodenum was very low, while its transit through the distal parts (jejunum and ileum) resulted in an increase in the pathogen population. The production of Shiga toxins by this enterohemorrhagic microorganism in the ileal effluents of the in vitro system was too low to be detected by ELISA assays. On the contrary, the three lactobacilli species assayed showed a considerable resistance to the gastric digestion, but not to the intestinal one, which affected their survival, and was especially evident in the case of both $L$. pentosus strains. In spite of this, high population levels for all assayed microorganisms were recovered at the end of the gastrointestinal passage. The results obtained in the present study show the potential use of table olives as a vehicle of beneficial microorganisms to the human body, as well as the need for good hygienic practices on the part of olive manufacturers in order to avoid the possibility of contamination by food-borne pathogens.

Keywords: food carrier, lactobacilli, pathogen, probiotic, survival, Shiga toxins, table olives, TIM system

\section{INTRODUCTION}

Green Spanish-style table olives are considered ready-to-eat products which are directly consumed without any prior cooking, and often, without any pasteurization or sterilization treatment. This makes olives a splendid vehicle of microorganisms (beneficial or harmful) to the human body. Thereby, the analysis of the microbiota adhered to the fruits acquires a relevant importance, although, incomprehensibly, this point has received scarce attention by researchers. Fermented olives contain biofilm structures formed mainly by lactic acid bacteria (LAB) and yeasts, which can reach population levels up to $8 \log _{10} \mathrm{CFU} \mathrm{g^{-1 }}$ in the epidermis of the fruits (Nychas etal., 2002; Arroyo-López etal., 2012; DomínguezManzano etal., 2012). Native LAB or yeast strains isolated from olive microbiota which show potential probiotic characteristics could be used as starters either to initiate fermentation or to obtain a functional product after adhesion to the fruits (Rodríguez-Gómez etal., 2013; Blana etal., 2014). To exert their health effect, microorganisms to be evaluated as probiotics should be able to survive the harsh conditions found in the human digestive tract such as acidic $\mathrm{pH}$ or high concentrations of digestive enzymes and bile salts.
Regarding safety issues, it is necessary to provide more knowledge about the behavior of pathogens in ready-to-eat fermented olives for future risk assessments. Enterohemorrhagic Escherichia coli is a major food-borne pathogen that causes hemorrhagic colitis and a life-threatening sequelae, the hemolytic uremic syndrome (Pennington, 2010). The behavior of the enterohemorrhagic E. coli O157:H7 during olive processing (fermentation and packing) has been reported by several authors (Spyropoulou et al., 2001; Skandamis and Nychas, 2003; Argyri et al., 2013; Grounda et al., 2013) but there is hitherto no available information about the survival of this pathogen in the case of a hypothetical intake of contaminated olives. To cause human illness, $E$. coli O157:H7 should not only survive its passage through the gastrointestinal tract but also coordinate the expression of virulence genes especially that of encoding Shiga toxins (Foster, 2013).

Most of the available data on probiotic or pathogen survival in the human digestive tract has been obtained in static in vitro systems that are not representative of the continuously changing variable during the gastrointestinal transit. The TNO gastrointestinal tract model (TIM system, Zeist, Netherlands) is an alternative dynamic multi-compartmental in vitro system which currently allows the closest simulation of in vivo physiological processes 
occurring within the stomach and small intestine of humans (Minekus et al., 1995; Guerra et al., 2012).

In this work, we used the TIM system to assess the survival and toxin production of an enterohemorrhagic E. coli strain inoculated in fermented olives during its transit through the upper human gastrointestinal tract. The survival of two potential probiotic strains isolated from table olives and belonging to Lactobacillus pentosus species (never studied before in dynamic digestive assays) was also evaluated and compared to that of a well-recognized probiotic microorganism (L. rhamnosus GG).

\section{MATERIALS AND METHODS OLIVES AND MICROORGANISMS}

Fermented olives of the Manzanilla variety (Olea europaea pomiformis) were used in the present study. The fruits were previously fermented for two months according to the Spanish-style (Garrido-Fernández etal., 1997) and then pasteurized at $80^{\circ} \mathrm{C}$ for $15 \mathrm{~min}$ to avoid any microbial presence on the olive surface. Twenty-five grams of pasteurized olives were then homogenized with $250 \mathrm{~mL}$ of sterile water and independently inoculated with aerobic cultures of the reference strain E. coli O157:H7 EDL 933 ( $\mathrm{LB}, 24 \mathrm{~h}, 37^{\circ} \mathrm{C}$ ), the probiotic L. rhamnosus $\mathrm{GG}$, and the olive isolates L. pentosus TOMC-LAB2 and L. pentosus TOMC-LAB4 (MRS broth, $24 \mathrm{~h}, 37^{\circ} \mathrm{C}$ ) to reach a final population in the mix of 8.2, 9.1, 9.7, and $9.4 \log _{10} \mathrm{CFU}$, respectively. Except for the pathogen strain, these inoculation levels are similar for the lactic acid population usually present in $25 \mathrm{~g}$ of fermented olives. The two L. pentosus strains, originally isolated from Spanish-style table olive fermentations and belonging to the table olive microorganisms collection (TOMC) from Food Biotechnology Department of Instituto de la Grasa (CSIC-Seville), were selected because of their previously described probiotic characteristics (Bautista-Gallego et al., 2013) and good performance as starter in previous fermentation trials carried out at laboratory scale (Rodríguez-Gómez et al., 2013). Table 1 shows the main physicochemical conditions of the fermented olives at the moment of introduction into the TIM system.

\section{SIMULATED HUMAN DIGESTIVE CONDITIONS}

The TIM system, which simulates the physiological processes occurring in the stomach, duodenum, jejunum, and ileum, was programmed to reproduce the digestion of a solid food matrix in a healthy human adult using the protocol described by EtienneMesmin et al. (2011). The total duration of the digestions was 300 min with $n=2$ digestions for each different microorganism. The parameters used for in vitro digestion are described in Table 2. Samples were taken in the test meal (initial intake) after inoculation and before its introduction into the artificial stomach, and regularly collected during digestion in the different compartments of the system (stomach, duodenum, jejunum, and ileum) as well as in the cumulative ileal effluents (CIE). Microbial counting was performed on LB agar (for E. coli) or on MRS agar (for the lactobacilli strains; Oxoid LTD, Basingstoke, Hampshire, England). Because food and microorganisms are in continuous transit from one compartment to the next, in order to assess microbial survival rates in the TIM system, control digestions $(n=2)$ were carried out with the same protocol conditions used for microorganisms, but with water containing only $0.8 \%(\mathrm{w} / \mathrm{v})$ of blue dextran (Minekus et al., 1995). This compound is a non absorbable transit marker, which will represent a $100 \%$ survival percentage for bacteria. Thereby, curves below the transit marker will represent the mortality of the microorganism, while curves above the transit marker will be indicative of bacteria growth renewal.

\section{DETERMINATION OF THE PRODUCTION OF SHIGA TOXINS BY THE $E$. coli STRAIN}

Shiga toxins produced by E. coli O157:H7 EDL 933 in the ileal effluents of the TIM system were dosed by enzyme linked immunosorbent assay (ELISA) using the Ridascreen ${ }^{\circledR}$ Verotoxin kit (R-Biopharm, Darmstadt, Germany). Purified toxin Stx2 (Toxin Technology ${ }^{\circledR}$, Sarasota, FL, USA) was used to establish standard calibration curves. Samples were diluted to half with the provided diluent and the dosage was made according to the manufacturer's instructions. Each digestive sample was analyzed in duplicate and serial dilutions of purified toxin were analyzed in triplicate.

\section{STATISTICAL ANALYSIS}

Significant differences among treatments $(p \leq 0.05)$ were tested by analysis of variance (ANOVA) followed by a Fisher-LSD post hoc comparison test carried out with STATISTICA 7.0 software package (Statsoft Inc, Tulsa, OK, USA).

\section{RESULTS AND DISCUSSION}

In the present study, fermented olives were independently inoculated with a food-borne pathogen and three potential beneficial microorganisms in order to determine their survival during their passage through the upper gastrointestinal tract of humans and the pathogen's ability to produce toxins. So far, there is no information available on the survival of microorganisms through the different compartments of the human gastrointestinal tract using table olives as the food matrix. The only related study was carried out by Lavermicocca et al. (2005), who fed volunteers with olives inoculated with the human origin strain $L$. paracasei IMPC2.1. These authors were able to recover the mentioned microorganism from fecal samples, but they did not obtain information on its resistance to specific gastric and intestinal conditions.

The behavior of the major food borne pathogen E. coli O157:H7 (strain EDL 933) during its transit through the different compartments of the TIM system is shown in Figure 1. In the stomach and duodenum, its population decreased considerably, probably due to the occurrence of stringent conditions such as gastric acidity, digestive enzymes, or bile salts (Smith, 2003). The highest cell mortality was observed in the artificial stomach from 90 min onward, when the $\mathrm{pH}$ fell below 3 units (see Table 2). At this time, the population decreased by more than $2 \log _{10}$ CFU compared to the transit marker used as control, and no living cells were recovered from this compartment at $150 \mathrm{~min}$. In the duodenum, at $180 \mathrm{~min}$, around $3 \log _{10}$ CFU were lost compared to the marker.

On the contrary, in the distal parts of the artificial gastrointestinal tract (jejunum and ileum), E. coli O157:H7 resumption was observed especially at the end of digestion. Thereby, at time $300 \mathrm{~min}$, the counts for bacteria even exceeded that of 
Table 1 | Experimental design and physicochemical conditions of the fermented olives used in the present work as food matrix.

\begin{tabular}{llll}
\hline $\begin{array}{l}\text { Reference for } \\
\text { treatment }\end{array}$ & Inoculated microorganism & $\begin{array}{l}\text { Attributed effects on } \\
\text { human health }\end{array}$ & $\begin{array}{l}\text { Physicochemical conditions of } \\
\text { fermented olives* }\end{array}$ \\
\hline Ec & Escherichia coli O157:H7 & Enterohemorrhagic & $\mathrm{pH}=4.1$ \\
$\mathrm{ED}$ & pathogen 933 & Palt $=50.0 \mathrm{~g} / \mathrm{l}$ \\
$\mathrm{Lp2}$ & Lactobacillus rhamnosus GG & Potential probiotic strains & Sugars $\leq 2.0 \mathrm{~g} / \mathrm{l}$ \\
$\mathrm{Lp} 4$ & L. pentosus LAB2 & Phenolic compounds $=900 \mathrm{mg} / \mathrm{kg}$ \\
\hline
\end{tabular}

*Fruits had an average size of $4.06 \pm 0.49 \mathrm{~g}$ and area surface of $10.93 \pm 1.07 \mathrm{~cm}^{2}$.

Table 2 | Parameters of gastrointestinal digestion in the TIM system when simulating digestive conditions of a healthy adult after intake of a solid food matrix.

\begin{tabular}{|c|c|c|c|c|c|}
\hline Compartment & Vol (ml) & $\mathrm{pH} /$ time (min) & Secretion & $t_{1 / 2}(\min )$ & $\beta$ coefficient \\
\hline Stomach & 300 & $\begin{array}{l}2 / 0,6 / 5,5.7 / 15 \\
4.5 / 45,2.9 / 90,2.3 / 120 \\
1.8 / 240,1.6 / 300\end{array}$ & $\begin{array}{l}0.25 \mathrm{ml} / \mathrm{min} \text { of pepsin }(2080 \mathrm{IU} / \mathrm{ml}), 0.25 \mathrm{ml} / \mathrm{min} \text { of lipase } \\
(250.5 \mathrm{IU} / \mathrm{ml}), 0.25 \mathrm{ml} / \mathrm{min} \text { of } \mathrm{HCl}(1.5 \mathrm{M}) \text { if necessary. }\end{array}$ & 85 & 1.8 \\
\hline Duodenum & 30 & Maintained at 6.0 & $\begin{array}{l}0.5 \mathrm{ml} / \mathrm{min} \text { of bile salts }(4 \% \text { during the first } 30 \mathrm{~min} \text { of } \\
\text { digestion and then } 2 \%), 0.25 \mathrm{ml} / \mathrm{min} \text { of pancreatic juice } \\
\left(10^{3} \mathrm{USP} / \mathrm{ml}\right), 0.25 \mathrm{ml} / \mathrm{min} \text { of intestinal electrolyte } \\
\text { solution, } 0.25 \mathrm{ml} / \mathrm{min} \text { of } \mathrm{NaHCO}_{3}(1 \mathrm{M} \text { ) if necessary, } \\
23,600 \mathrm{IU} \text { of trypsin (at the beginning of digestion). }\end{array}$ & & \\
\hline Jejunum & 130 & Maintained at 6.9 & $0.25 \mathrm{ml} / \mathrm{min}$ of $\mathrm{NaHCO}_{3}(1 \mathrm{M})$ if necessary. & & \\
\hline Ileum & 130 & Maintained at 7.2 & $0.25 \mathrm{ml} / \mathrm{min}$ of $\mathrm{NaHCO}_{3}(1 \mathrm{M})$ if necessary. & 250 & 2.5 \\
\hline
\end{tabular}

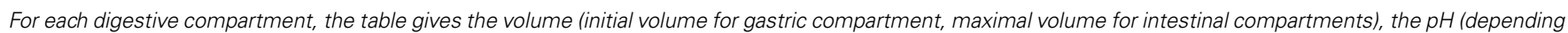

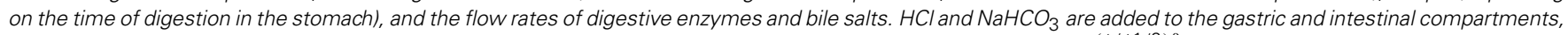

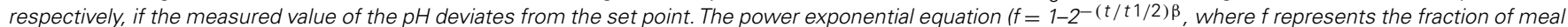

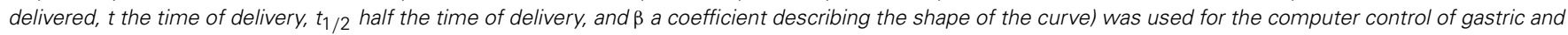
ileal deliveries into the TIM system. Parameters used for $t_{1 / 2}$ and $\beta$ for gastric and ileal deliveries are mentioned in the table.

the transit marker, chiefly in the jejunum (increase of $0.5 \log _{10}$ CFU compared to the control marker). At $300 \mathrm{~min}$, a high amount of viable cells were recovered from the ileal effluents, decreasing only by $0.2 \log _{10}$ CFU with respect to the blue dextran. Bacterial growth renewal has been previously observed in the distal compartments of the TIM system for other E. coli strains (Ganzle et al., 1999; Etienne-Mesmin et al., 2011; Miszczycha et al., 2014). This growth resumption was probably linked to less stringent environmental conditions, such as a $\mathrm{pH}$ closer to neutrality, lower concentrations of bile salts (owing to their passive reabsorption in the TIM system), and/or an increase in the residence time of bacteria. This event led to an increase in the number of bacteria which could potentially enter into the colon and may potentiate the harmfulness of the food-borne pathogen in the hypothetical case of an intake of contaminated olives.

Large variations in survival rates have been obtained for $E$. coli $\mathrm{O} 157: \mathrm{H7}$ in diverse in vitro digestion assays (Arnold and Kaspar, 1995; Takumi et al., 2000; Foster, 2004; Tamplin, 2005; Etienne-Mesmin et al., 2011). This wide range of response may be explained by differences between culture conditions, digestive systems (static or dynamic) and parameters, food matrices and also bacterial strains. Interestingly, the only studies that evaluated the behavior of E. coli O157:H7 in dynamic conditions gave survival rates after gastric digestion close to those obtained in the present work (Takumi et al., 2000; Etienne-Mesmin et al., 2011; Miszczycha et al., 2014). In table olive packing, this microorganism has shown also a considerable survival being present until 19th days of storage on olive fruits at low $\mathrm{pH}$ (4.2) and high salt concentration (60 g/l; Argyri et al., 2013).

Hitherto, there is no available data regarding the production (site and amount) of Shiga toxins by enterohemorrhagic E. coli strains during their transit through the human gastrointestinal tract. This study is the first to investigate toxin production by this food-borne pathogen in a human simulated digestive environment. Toxin levels in the ileal effluents of the TIM system were too low to be quantified by ELISA assays, which had a detection threshold value of $0.31 \mathrm{ng} / \mathrm{ml}$ (data not shown), indicating that under the tested conditions the pathogen was not able to produce Shiga toxins, its main virulence factor, at least at sufficient levels to be detected. It also suggests that toxin production, if it occurs, would rather 

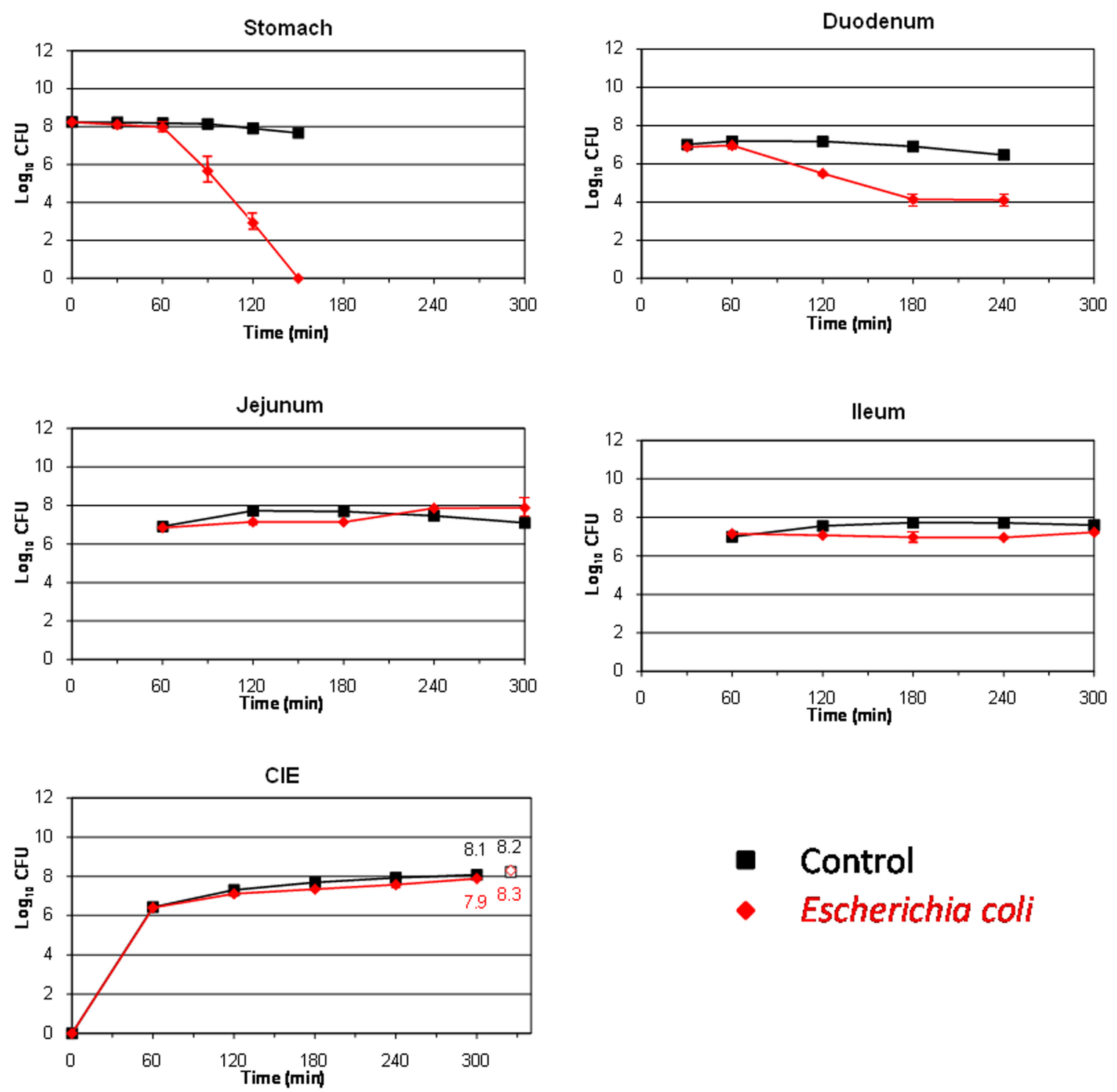

FIGURE 1 | Survival (plate counts versus time) of the food-borne pathogen Escherichia coli 0157:H7 EDL 933 in the different compartments and ileal effluents (CIE) of the TIM system compared to a non absorbable transit marker, blue dextran, used as control. Results are expressed as log 10 cfu \pm SDs $(n=2)$.

take place in the colon, which is already described as the main site of pathogenicity for EHEC strains (Shigeno etal., 2002).

This study also aims to assess the survival of two L. pentosus strains isolated from table olives for their use as putative probiotics. LAB species are well known for their ability to resist and live in many different acidified fermented vegetables, among them table olives (Hurtado et al., 2012). The L. pentosus strains assayed in this work were originally isolated from table olive fermentations, so, presumably, they should be well adapted to the acidic environment which governs this type of product. Figures 2 and $\mathbf{3}$ show the results obtained for the survival in the TIM system of L. pentosus TOMC-LAB2 and TOMC-LAB4, respectively. Both bacteria showed a considerable resistance to gastric digestion with a slight decrease at time 150 min of less than $0.1 \log _{10}$ CFU with respect to the transit marker. However, their survival through the duodenum was considerably lower, especially evident in the case of the L. pentosus TOMCLAB2 strain with a reduction of more than $3 \log _{10}$ CFU at $240 \mathrm{~min}$. On the contrary, the survival of neither microorganism was affected by the conditions encountered in the distal parts of the gastrointestinal tract (jejunum and ileum). At $300 \mathrm{~min}$, compared to the transit marker, the amount of bacteria recovered from the ileal effluents was reduced by 2.1 and $1.3 \log _{10}$ CFU for L. pentosus TOMC-LAB2 and TOMC-LAB4 strains, respectively. 

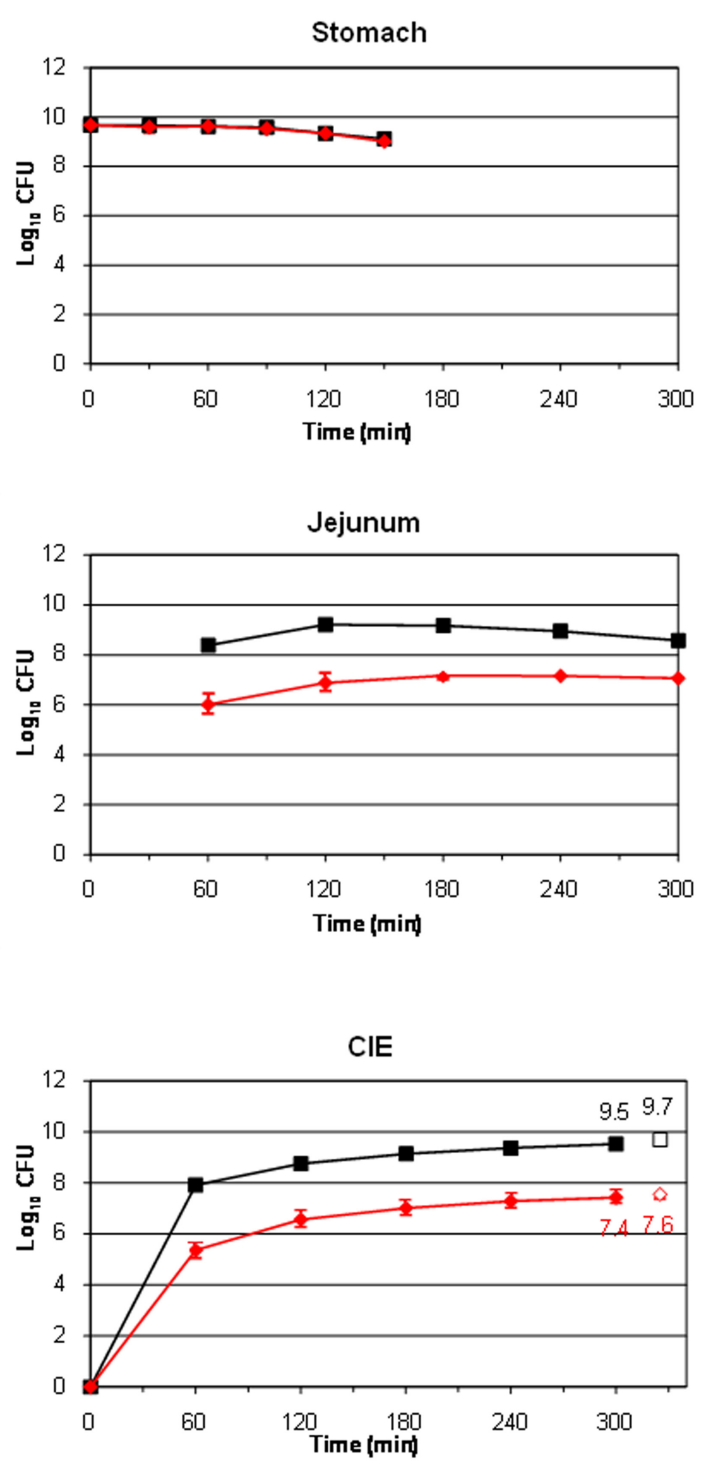
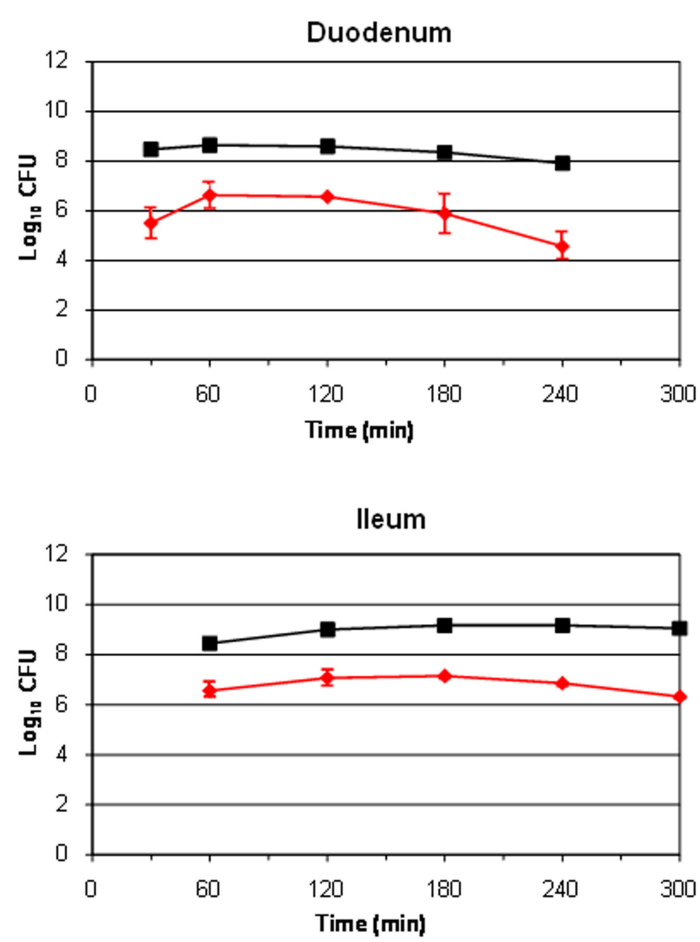

- Control

- Lactobacillus pentosus LAB2

FIGURE 2 | Survival (plate counts versus time) of the potential probiotic strain Lactobacillus pentosus TOMC-LAB2, isolated originally from table olives, in the different CIE of the TIM system compared to a non absorbable transit marker, blue dextran, used as control. Results are expressed as $\log _{10} \mathrm{cfu} \pm \mathrm{SDs}(n=2)$.

To be effective and confer health benefits on the host, probiotics must be able to survive passage through the human stomach and small intestine and be present in sufficient number to colonize the colonic environment (Del Piano et al., 2006). The survival of the $L$. pentosus strains isolated from table olives was compared to that of the well-known probiotic L. rhamnosus GG (Figure 4). This microorganism was slightly affected during its transit through the stomach (only $0.1 \log _{10}$ CFU reduction at time $150 \mathrm{~min}$ ). Pitino et al. (2012) also found a high resistance of this species to stomach digestion in a dynamic gastric model. On the contrary, the population decreased in higher proportions at the end of its transit through the duodenum $\left(\sim 1 \log _{10} \mathrm{CFU}\right.$ at time $\left.240 \mathrm{~min}\right)$ and it seemed to be not affected by the conditions occurring in the jejunum and ileum (no additional mortality was observed in either compartment). At $300 \mathrm{~min}$, high levels for this LAB species were recovered from the ileal effluents, decreasing by only $0.3 \log _{10}$ CFU compared to the transit marker.

Table 3 shows the percentage of survival obtained for the four assayed bacteria after their transit through the different compartments of the TIM system as well as in the ileal effluents at the end of digestion. As can be clearly deduced even though E. coli O157:H7 is considered a highly acid resistant pathogen (Foster, 2004), this microorganism was the most affected bacteria by gastric digestion, with statistically significant differences $(p<0.05)$ compared to lactobacilli strains. L. rhamnosus $G G$ was the most resistant bacteria during its transit through duodenum, although without 

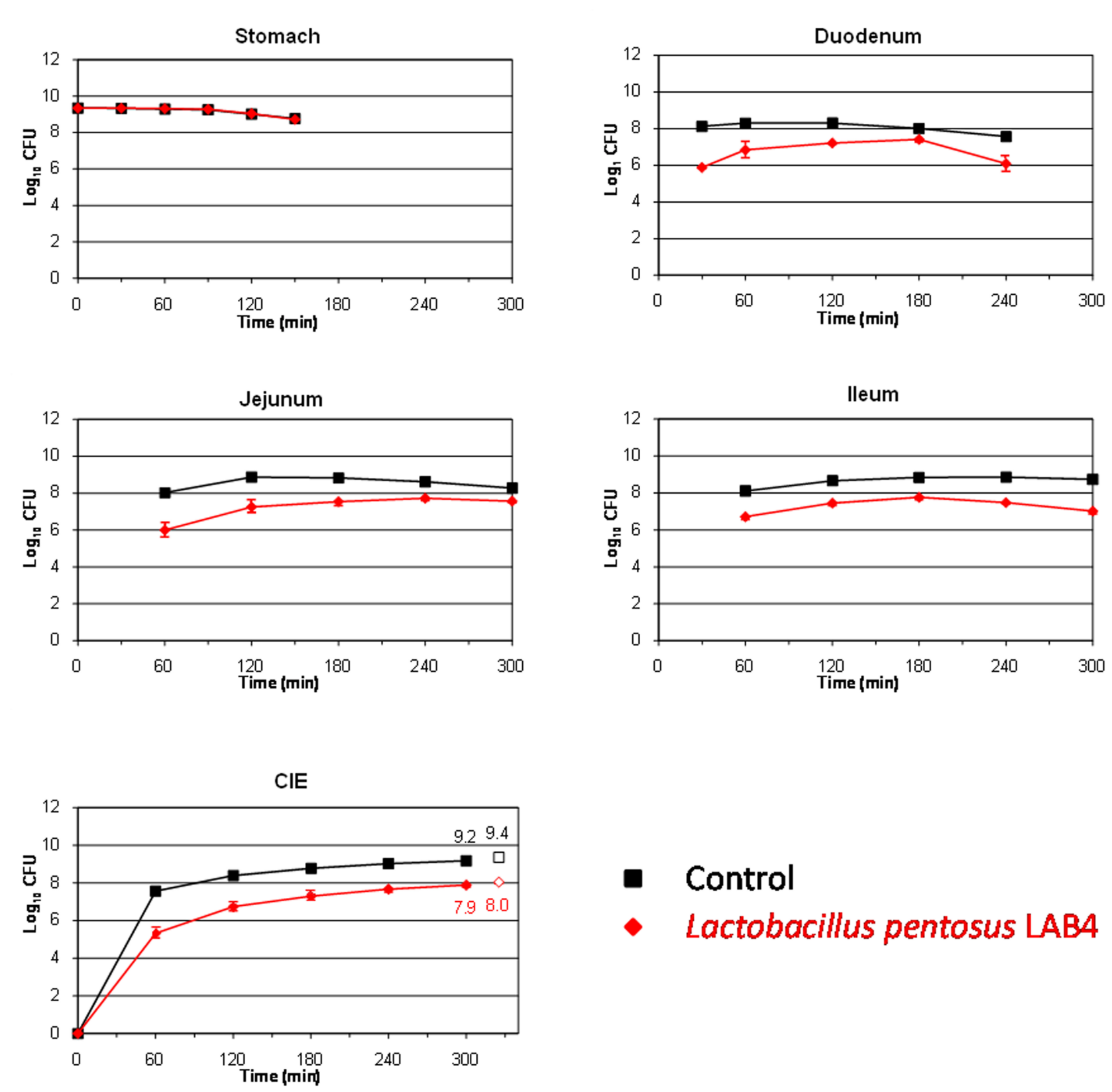

Control

\section{Lactobacillus pentosus LAB4}

FIGURE 3 | Survival (plate counts versus time) of the potential probiotic strain L. pentosus TOMC-LAB4, isolated originally from table olives, in the different CIE of the TIM system compared to a non absorbable transit marker, blue dextran, used as control. Results are expressed as log $10 \mathrm{cfu} \pm \mathrm{SDs}$ $(n=2)$.

significant differences according to a Fisher-LSD post hoc comparison test. This is in agreement with previous results obtained by Silva et al. (1987) and Pitino et al. (2012) who cataloged L. rhamnosus as an acid and bile-resistant species. Bile salts are described as toxic at high concentrations for bacterial cells by disorganizing the lipid bi-layer structure of the cellular membranes (Thanassi et al., 1997). This is presumably the reason why all assayed microorganisms reduced their population during their transit through the duodenum. On the contrary, the food-borne pathogen was the most adapted microorganism in the distal parts of the gastrointestinal tract (jejunum and ileum), with significant differences $(p<0.05)$ compared to the lactobacilli species in the ileum. Thereby, after gastric and small intestinal transit, the survival rate was statistically higher for E. coli O157:H7 EDL 933 (117.5\%) than for the rest of bacteria (from 0.7 to $40.5 \%$ ), indicating that a higher percentage of the pathogen population could enter into the colon compared to the lactobacilli species in the case of a hypothetical intake of contaminated olives. L. pentosus TOMC-LAB2 

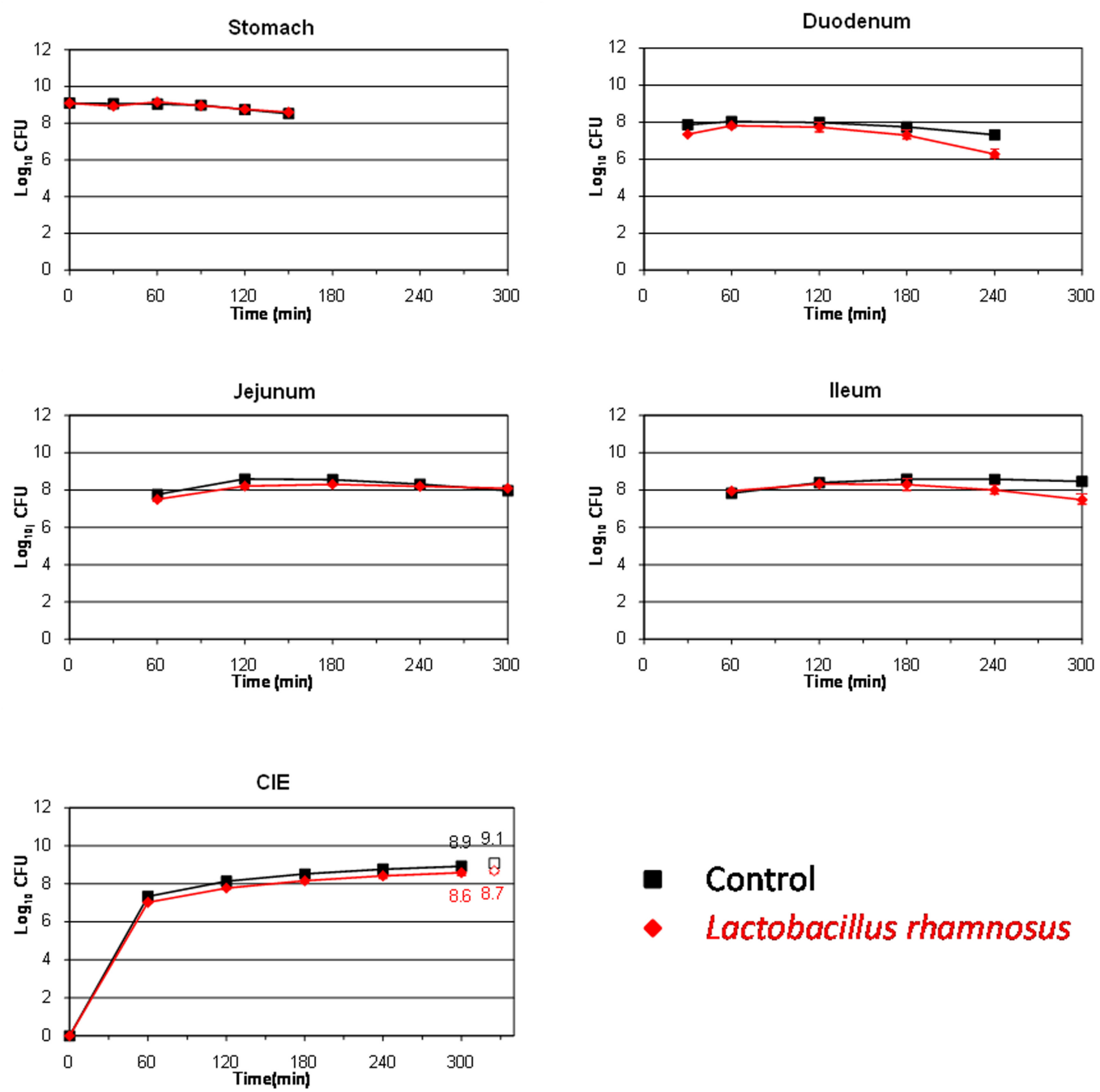

FIGURE 4 | Survival (plate counts versus time) of the probiotic strain L. rhamnosus GG in the different CIE of the TIM system compared to a non absorbable transit marker, blue dextran, used as control. Results are expressed as $\log _{10} \mathrm{cfu} \pm \operatorname{SDs}(n=2)$.

Table 3 | Survival (\%) of the four tested strains at the end of in vitro digestions in the different compartments and ileal effluents (CIE) of the TIM system, using fermented Manzanilla olives as food matrix.

\begin{tabular}{|c|c|c|c|c|c|c|}
\hline Microorganism & $E_{150}$ & $D_{240}$ & $\mathbf{J}_{300}$ & $\mathrm{I}_{300}$ & $\mathrm{ClE}_{300}$ & $\mathrm{TF}_{325}$ \\
\hline E. coli O157:H7 EDL 933 & $0.00(0.00)^{a}$ & $0.01(0.00)^{\mathrm{a}}$ & $11.55(8.69)^{\mathrm{a}}$ & $45.27(4.45)^{\mathrm{a}}$ & $45.45(11.66)^{a}$ & $117.47(26.68)^{\mathrm{a}}$ \\
\hline L. rhamnosus GG & $32.80(3.47)^{\mathrm{c}}$ & $0.16(0.05)^{\mathrm{a}}$ & $2.52(0.01)^{a}$ & $10.97(4.55)^{b}$ & $31.29(5.91)^{a}$ & $40.48(7.37)^{\mathrm{c}}$ \\
\hline L. pentosus LAB2 & $18.13(0.05)^{b}$ & $0.001(0.001)^{a}$ & $0.05(0.00)^{\mathrm{a}}$ & $0.24(0.02)^{c}$ & $0.55(0.13)^{\mathrm{b}}$ & $0.74(0.14)^{\mathrm{b}}$ \\
\hline L. pentosus LAB4 & $24.11(5.22)^{\mathrm{b}, c}$ & $0.07(0.05)^{a}$ & $0.47(0.06)^{\mathrm{a}}$ & $1.69(0.66)^{c}$ & $3.47(1.03)^{\mathrm{b}}$ & $4.91(1.04)^{b, c}$ \\
\hline
\end{tabular}

$E_{150}, D_{240}, J_{300}$ and $l_{300}$ stands for instantaneous percentages in the stomach (150 min), duodenum (240 min), jejunum (300 min), and ileum (300 min), respectively. $\mathrm{CIE}_{300}$ and $\mathrm{TF}_{325}$ stands for cumulative percentages from ileal effluents (0-300 min) and cumulative percentages from ileal effluents (0-300 min) plus final gastrointestinal residue, respectively. SD in parentheses. Values followed by different superscript letters, within the same column, are significantly different ( $p<0.05)$ according to a Fisher-LSD post hoc comparison test.

and TOMC-LAB4 have already shown promising probiotic characteristics (Bautista-Gallego et al., 2013) as well as a considerable capacity for adhesion to the olive epidermis (Arroyo-López et al.,
2012). Population levels higher than $7.5 \log _{10}$ CFU were recovered for both strains after their transit through the artificial ileum and before entering into the colon (survival percentages of 0.6 
and $3.5 \%$ for L. pentosus TOMC-LAB2 and TOMC-LAB4, respectively), compared to $8.5 \log _{10}$ CFU obtained for L. rhamnosus GG (survival percentage of $31.3 \%$ ). This makes them good candidates for use as probiotic agents, which could increase the functional value of table olives.

\section{CONCLUSION}

In summary, these results encourage further research on the development of table olives as a functional food, because, apparently, it is a good vehicle of microorganisms to the human body. Nevertheless, at the same time, they also show the necessity of good hygienic practices by olive manufacturers to avoid any possibility of intake of EHEC-contaminated olives, even though, apparently, the pathogen was not able to produce toxins during its transit through the upper human gastrointestinal tract following olive intake.

\section{ACKNOWLEDGMENTS}

The research leading to these results has received funding from the EU's Seventh Framework Program (FP7/2007-2013) under grant agreement $n^{\circ} 243471$ (PROBIOLIVES). Francisco N. ArroyoLópez wish to express his gratitude to the Spanish Government for his Ramón y Cajal postdoctoral research contract, as well as CSIC for financial support of the research mobility program in foreign countries (A12012PM2-PA1003309). Verónica RomeroGil thanks to the Agrifood Campus of International Excellence (ceiA3) for her predoctoral fellowship (program of formation of Ph.D. in companies).

\section{REFERENCES}

Argyri, A. A., Lyra, E., Panagou, E. Z., and Tassou, C. C. (2013). Fate of Escherichia coli $\mathrm{O} 157: \mathrm{H} 7$, Salmonella Enteritidis and Listeria monocytogenes during storage of fermented green table olives in brine. Food Microbiol. 36, 1-6. doi: 10.1016/j.fm.2013.04.001

Arnold, K. W., and Kaspar, C. W. (1995). Starvation and stationary-phase induced acid tolerance in Escherichia coli O157:H7. Appl. Environ. Microbiol. 61, 20372039.

Arroyo-López, F. N., Bautista-Gallego, J., Domínguez-Manzano, J., Romero-Gil, V., Rodriguez-Gómez, F., García-García, P., et al. (2012). Formation of lactic acid bacteria-yeasts communities on the olive surface during Spanish-style Manzanilla fermentations. Food Microbiol. 32, 295-301. doi: 10.1016/j.fm.2012.07.003

Bautista-Gallego, J., Arroyo-López, F. N., Rantsiou, K., Jiménez Díaz, R., Garrido Fernández, A., and Cocolin, L. (2013). Screening of lactic acid bacteria isolated from fermented table olives with probiotic potential. Food Res. Int. 50, 135-142. doi: 10.1016/j.foodres.2012.10.004

Blana, V. A., Grounta, A., Tassou, C. C., Nychas, G. J., and Panagou, E. Z. (2014). Inoculated fermentation of green olives with potential probiotic Lactobacillus pentosus and Lactobacillus plantarum starter cultures isolated from industrially fermented olives. Food Microbiol. 38, 208-218. doi: 10.1016/j.fm.2013.09.007

Del Piano, M., Morelli, L., Strozzi, G. P., Allesina, S., Barba, M., Deidda, F., et al. (2006). Probiotics: from research to consumer. Dig. Liver Dis. 38, 248-255. doi: $10.1016 /$ S1590-8658(07)60004-8

Domínguez-Manzano, J., León-Romero, Á., Olmo-Ruiz, C., Bautista-Gallego, J., Arroyo-López, F. N., Garrido-Fernández, A., et al. (2012). Biofilm formation on abiotic and biotic surfaces during Spanish style green table olive fermentation. Int. J. Food Microbiol. 157, 230-238. doi: 10.1016/j.ijfoodmicro.2012.05.011

Etienne-Mesmin, L., Livrelli, V., Privat, M., Denis, S., Cardot, J. M., Alric, M., et al. (2011). Effect of a new probiotic Saccharomyces cerevisiae strain on survival of Escherichia coli $\mathrm{O} 157: \mathrm{H7}$ in a dynamic gastrointestinal model. Appl. Environ. Microbiol. 77, 1127-1131. doi: 10.1128/AEM.02130-10

Foster, D. B. (2013). Modulation of the enterohemorrhagic E. coli virulence program through the human gastrointestinal tract. Virulence 4, 315-323. doi: 10.4161/viru. 24318
Foster, J. W. (2004). Escherichia coli acid resistance: tales of an amateur acidophile. Nat. Rev. Microbiol. 2, 898-907. doi: 10.1038/ nrmicro1021

Ganzle, M. G., Hertel, C., Van der Vossen, J. M., and Hammes, W. P. (1999). Effect of bacteriocin-producing Lactobacilli on the survival of Escherichia coli and Listeria in a dynamic model of the stomach and the small intestine. Int. J. Food Microbiol. 48, 21-35. doi: 10.1016/S0168-1605(99)00025-2

Garrido-Fernández, A., Fernández Díez, M. J., and Adams, R. M. (1997). Table Olives. Production and Processing. London: Chapman \& Halls. doi: 10.1007/9781-4899-4683-6

Grounda, A., Nychas, G. J. E., and Panagou, E. Z. (2013). Survival of foodborne pathogens on natural black table olives after post-processing contamination. Int. J. Food Microbiol. 161, 197-202. doi: 10.1016/j.ijfoodmicro.2012. 12.017

Guerra, A., Etienne-Mesmin, L., Livrelli, V., Denis, S., Blanquet-Diot, S., and Alric, M. (2012). Relevance and challenges of in vitro gastrointestinal models in simulating human digestion. Trends Biotechnol. 30, 591-600. doi: 10.1016/j.tibtech.2012.08.001

Hurtado, A., Reguant, C., Bordons, A., and Rozès, N. (2012). Lactic acid bacteria from fermented table olives. Food Microbiol. 31, 1-8. doi: 10.1016/j.fm.2012.01.006

Lavermicocca, P., Valerio, F., Lisa Lonigro, S., De Angelis, M., Morelli, L., Callegari, M. L., et al. (2005). Study of adhesion and survival of Lactobacilli and Bifidobacteria on table olives with the aim of formulating a new probiotic food. Appl. Environ. Microbiol. 71, 4233-4240. doi: 10.1128/AEM.71.8.42334240.2005

Minekus, M., Marteau, P., Havenaar, R., and Huis in't Veild, J. H. (1995). A multicompartmental dynamic computer-controlled model simulating the stomach and small intestine. ATLA 23, 197-209.

Miszczycha, S. D., Thévenot, J., Denis, S., Callon, C., Livrelli, V., Alric, M., et al. (2014). Survival of Escherichia coli O26:H11 exceeds that of Escherichia coli $\mathrm{O} 157: \mathrm{H7}$ as assessed by simulated human digestion of contaminated raw milk cheeses. Int. J. Food Microbiol. 172, 40-48. doi: 10.1016/j.ijfoodmicro.2013. 11.029

Nychas, G. J. E., Panagou, E. Z., Parker, M. L., Waldron, K. W., and Tassou, C. C. (2002). Microbial colonization of naturally black olives during fermentation and associated biochemical activities in the cover brine. Lett. Appl. Microbiol. 34, 173-177. doi: 10.1046/j.1472-765x.2002. 01077.x

Pennington, H. (2010). Escherichia coli O157. Lancet 376, 1428-1435. doi: 10.1016/S0140-6736(10)60963-4

Pitino, I., Randazzo, C. L., Cross, K. L., Parker, M. L., Bisignano, C., Wickham, M. S. J., et al. (2012). Survival of Lactobacillus rhamnosus strains inoculated in cheese matrix during simulated human digestion. Food Microbiol. 31, 57-63. doi: 10.1016/j.fm.2012.02.013

Rodríguez-Gómez, F., Bautista-Gallego, J., Arroyo-López, F. N., Romero-Gil, V., Jiménez-Díaz, R., Garrido-Fernández, A., et al. (2013). Table olive fermentation with multifunctional Lactobacillus pentosus strains. Food Control 34, 96-105. doi: 10.1016/j.foodcont.2013.04.010

Shigeno, T., Akamatsu, T., Fujimori, K., Nakatsuji, Y., and Nagata, A. (2002). The clinical significance of colonoscopy in hemorrhagic colitis due to enterohemorrhagic Escherichia coli O157:H7 infection. Endoscopy 34, 311-314. doi: $10.1055 / \mathrm{s}-2002-23644$

Silva, M., Jacobus, N. V., Deneke, C., and Gorbach, S. L. (1987). Antimicrobial substance from a human Lactobacillus strain. Antimicrob. Agents Chemother. 31, 1231-1233. doi: 10.1128/AAC.31.8.1231

Skandamis, P. N., and Nychas, G. J. E. (2003). Modeling the microbial interaction and the death of Escherichia coli O157:H7 during the fermentation of Spanish style green table olives. J. Food Prot. 66, 1166-1175.

Smith, J. L. (2003). The role of gastric acid in preventing foodborne disease and how bacteria overcome acid conditions. J. Food Prot. 66, 1292-1303.

Spyropoulou, K. E., Chorianopoulos, N. G., Skandamis, P. N., and Nychas, G. J. E. (2001). Survival of Escherichia coli O157:H7 during the fermentation of Spanish-style green table olives (Conservolea variety) supplemented with different carbon sources. Int. J. Food Microbiol. 66, 3-11. doi: 10.1016/S0168-1605(00) 00510-9

Takumi, K., De Jonge, R., and Havelaar, A. (2000). Modelling inactivation of Escherichia coli by low $\mathrm{pH}$ : application to passage through the stomach of 
young and elderly people. J. Appl. Microbiol. 89, 935-943. doi: 10.1046/j.13652672.2000.01193.x

Tamplin, M. L. (2005). Inactivation of Escherichia coli O157:H7 in simulated human gastric fluid. Appl. Environ. Microbiol. 71, 320-325. doi: 10.1128/AEM.71.1.320325.2005

Thanassi, D., Cheng, G. W. L., and Nikaido, H. (1997). Active efflux of bile salts by Escherichia coli. J. Bacteriol. 179, 2512-2518.

Conflict of Interest Statement: The authors declare that the research was conducted in the absence of any commercial or financial relationships that could be construed as a potential conflict of interest.

Received: 23 July 2014; paper pending published: 26 August 2014; accepted: 29 September 2014; published online: 14 October 2014
Citation: Arroyo-López FN, Blanquet-Diot S, Denis S, Thévenot J, Chalancon S, Alric M, Rodríguez-Gómez F, Romero-Gil V, Jiménez-Díaz R and Garrido-Fernández A (2014) Survival of pathogenic and lactobacilli species of fermented olives during simulated human digestion. Front. Microbiol. 5:540. doi: 10.3389/fmicb.2014.00540 This article was submitted to Food Microbiology, a section of the journal Frontiers in Microbiology.

Copyright (c) 2014 Arroyo-López, Blanquet-Diot, Denis, Thévenot, Chalancon, Alric, Rodríguez-Gómez, Romero-Gil, Jiménez-Díaz and Garrido-Fernández. This is an open-access article distributed under the terms of the Creative Commons Attribution License (CC BY). The use, distribution or reproduction in other forums is permitted, provided the original author(s) or licensor are credited and that the original publication in this journal is cited, in accordance with accepted academic practice. No use, distribution or reproduction is permitted which does not comply with these terms. 